surgery for locally recurrent disease, a figure which we would regard as higher than desirable.

Though the results of our studies and those of others may lead us to revise our approach in due course, we think that meantime we should continue to explain to our elderly patients that there are two forms of treatment available. One entails immediate surgery, the other a trial of tamoxifen surgery then being required only if the disease is not controlled, which is likely in less than half of those treated. The choice is theirs.

A P M FORREST

U CHETTY

D GASKELL

R A HAWKINS

University Department of Surgery,

Edinburgh EH3 9YW

\section{New Zealand cervical cancer study: could it happen again?}

Judge Sylvia Cartwright has now decreed that cervical carcinoma in situ (cervical intraepithelial neoplasia grade 3 ) should be construed legally as a potentially premalignant condition and patients afforded all active treatment rather than simple follow up. Dr Charlotte Paul then queries "Could it happen again?" (20 August, p 533).

$\mathrm{Mr} \mathrm{J}$ A Jordan has recently argued the necessity for a multicentre comparative study of cytology and colposcopy in the management of minor grades of cervical neoplasia (2 July, p 6). In addition, Drs David Jenkins and $\mathrm{M} \mathbf{H}$ Jones have given preliminary information on a multicentre comparative study of patients who have presented with mild dyskaryosis at the Whittington Hospital (20-27 August, p 555). Dr Jenkins correctly references worries that the biology of cervical cancer today may be far more aggressive than is generally realised and that low grade cytologica abnormality may be associated with areas of high grade cervical intraepithelial neoplasia.

The British Society for Clinical Cytology and the intercollegiate working party on cervical cytology screening have both recommended repeat smea tests at three to six months for mild dyskaryosis, with colposcopy and biopsy for persistent lesions. Most centres would then undertake some form of ablative therapy for any intraepithelial neoplasia present. Against, this background it is somewhat surprising to see that one of the groups of $\mathrm{Dr}$ Jenkins and Dr Jones is being managed by cytological surveillance alone. It would appear to be happening again.

DAVID SLATER

Rotherham District Hospital,

Rotherham S60 2UD

AUTHORS' REPLY,-Our worry about the biology of cervical cancer is equally matched by our concern about the apparent sharply contradictory evidence over the protection offered by properly organised cytological screening.

Our present study is retrospective and both groups are currently undergoing colposcopic examination with colpophotography and biopsy, including laser cone biopsy where appropriate. This is aimed at providing a detailed endpoint in the study of carefully matched groups of patients who have previously been managed by cytological surveillance alone or by colposcopy and treatment. The method of management was chosen according to the practice of the physician responsible for the patients' management two or more years ago Furthermore, every patient in the study is being offered treatment if required.

This study was carefully designed to circumvent the possible ethical and practical problems of prospective study and provide a rapid answer which clearly would not be available until after several years of follow up. We would, however, endorse the call for prospective trials, which should probably also include the full evaluation of the place of cervicographic triage and of the "see and treat" policy. We regard our present study as a necessary stage in preparing the way.

The report referred to by Dr Slater which recommended an interval of three to six months for repeat smears also urged in the same paragraph: "The urgent need of a cost-effectiveness trial in which women with smears showing mild dyskaryosis are randomly allocated to a group followed by repeat smears, compared with a group who are offered immediate colposcopy and biopsy." There are many centres that do not refer mild dyskaryosis for colposcopy unless it has worsened (as opposed to simply persisted for three to six months). There is also a wide variation between pathologists in deciding how to conduct cytological surveillance. ${ }^{2}$ We are experiencing no difficulty in finding patients who have had persistent mild dyskaryosis and who have not had colposcopy. Should it now be suggested that the centres responsible for managing these patients are acting unethically?

Dr Slater's letter has highlighted the ethical debate which must surround prospective study of precancer. Inappropriate comparison with the New Zealand study, where women with high grade premalignant change (carcinoma in situ) and high grade abnormal cytology were left untreated, should not cloud the issue of the proper study of women who have low grade smear abnormalities such as mild dyskaryosis or below. Unpublished work in our department (Jarmulowicz et al) shows that women with mild dyskaryosis and cervical intraepithelial neoplasia grade 3 have significantly smaller lesions than women with severe dyskaryosis and cervical intraepithelial neoplasia grade 3 . This may well reflect different biological potential

Current advice from the intercollegiate working party on the timing and subsequent management of repeat cervical smears can reflect only the shortage of information. The entire mild dyskaryosis debate will probably be resolved only by meticulously conducted prospective studies along the lines suggested in our original letter.

DAVID JENKINS MICHAEL JONES

Department of Histopathology and Cytopathology, Whittington Hospital,

London

1 Intercollegiate Working Party on Cervical Cytology Screening. Report. London: Roval College of Pathologists, 1987:14. 2 Jenkins D, Tay SK. Management of mildly abnormal cervical smears. Lancet $1987 ; \mathrm{i}: 748-9$

\section{Health and the ozone layer}

I share the general concerns of Professor Rona M Mackie and Dr Michael H Ryecroft (6 August, p 369) about the ozone layer but am less sure about their predictions of its effect on skin cancers. They mention, without reference, a hypothesis that "the risk of non-melanoma skin cancer rises steeply once a critical number of hours of exposure to ultraviolet B is reached" and conclude that the incidence of non-melanoma skin cancer may jump dramatically.

The most direct analogy to the effects of an increase in total dose of solar ultraviolet radiation is the differences in the rates of skin cancer at different latitudes. The incidence of nonmelanoma skin cancer shows a regular association with latitude, consistent with either a linear or an exponential model, over a wide range. ${ }^{\prime}$ A $10 \%$ increase in the dose of ultraviolet radiation (which might be produced by a $5 \%$ decrease in ozone) seems equivalent to a move of about $2^{\circ}$ of latitude (138 miles) towards the equator. There is no suggestion of a threshold effect.

Moreover, both epidemiological and experimental data suggest that non-melanoma skin cancer is related to lifetime dose, ${ }^{2}$ and so the change in ambient ultraviolet radiation values would show effects gradually over subsequent decades.

I see little evidence also of their assertion that the impact of a change in ultraviolet radiation values may have a more rapid effect on melanoma than on non-melanoma skin cancer. Again the comparison shows that the change in the incidence of melanoma with latitude is similar to, not greater than the change in incidence of non-melanoma cancers.' The associations of melanoma with short term, intermittent exposure to the sun and with burning in the sun do not necessarily mean that the effects of ozone depletion would be more noticeable. People who have had high exposure to the sun for example, from regular outdoor work - have, paradoxically, a similar or even lower risk of melanoma than those who have had lower exposure, suggesting that the relation of risk of melanoma to total dose is not linear and that the effect of an increase may be less dramatic at high exposures. ${ }^{3}$ Again the birth cohort effects that have been observed in melanoma suggest that any changes would be gradual and seen over many decades. ${ }^{+}$The observation that melanoma showed short term increases after years of maximum sunspot activity has been rendered questionable in interpretation as new data show that years of sunspot activity are accompanied by decreased rather than increased ultraviolet radiation at ground level, ${ }^{5}$ perhaps because increased solar ultraviolet radiation gives a disproportionate increase in stratopheric ozone, which results in a decrease in ultraviolet radiation received at ground level. Rates of melanoma have been rising rapidly over many decades ${ }^{4}$ despite the absence of any increase in measured ultraviolet radiation, showing that behavioural changes and perhaps as yet unrecognised non-solar factors have more important influences than changes in ambient ultraviolet radiation.

A loss of stratospheric ozone is likely to produce a gradual rather than a dramatic change in the incidence skin cancer, and the effects, particularly for melanoma, will be small relative to the differences that are produced by variations in individua subjects' exposure to the sun. This conclusion must not be used to dismiss the importance of dealing with the problem of the ozone layer, whose effects go well beyond those of skin cancer.

J MARK ELWOOD

Department of Community Medicine and Epidemiology, Queen's Medical Centre,

Nottingham NG7 2UH

1 Fears TR, Scotto J, Schneiderman MA. Skin cancer, melanoma and sunlight. Am f Public Health 1976;66:461-4.

2 Scotto J, Fraumeni JF Jr. Skin (other than melanoma). In Schottenfeld D, Fraumeni JF, eds. Cancer epidemiology and Schottenfeld D, Fraumeni JF, eds. Cancer epudemion

3 Elwood JM, Gallagher RP, Hill GB, Pearson JCG. Cutaneou melanoma in relation to intermittent and constant sun exposure- The Western Canada Melanoma Study. Int f Cancer posure - The West $1985 ; 35: 427-33$.
19.

4 Lee JAH. Melanoma and exposure to sunlight. Epidemiol Reo 1982:4:110-36.

5 Berger DS. Fluctuations and trends in environmental UV loads. In: Passchier WF, Bosnjakovic BFM, eds. Human exposure to ultraviolet radiation. Amsterdam: Elsevier, 1987:213-20.

\section{Fatal pulmonary aspiration of oral activated charcoal}

Over the past year and half we have used repeated doses of activated charcoal in the management of 15 patients who presented with acute poisoning 